\title{
ORIGINAL
}

\section{DISTRIBUCIÓN Y TENDENCIA DE LA TUBERCULOSIS POR GRUPOS DE EDADES Y POR MUNICIPIOS EN CIUDAD DE LA HABANA, CUBA. (1986-1998)}

\author{
Susana Borroto Gutiérrez (1), Luis Armas Pérez (1), Edilberto González Ochoa (1) Otto Peláez \\ Sánchez.(2) Ana Luisa Arteaga Yero (2) y José Sevy Court (2)
}

(1) Instituto de Medicina Tropical "Pedro Kourí". La Habana. Cuba

(2) Centro Provincial de Higiene y Epidemiología. Ciudad de La Habana.

\section{RESUMEN}

Fundamento: La provincia Ciudad de la Habana, capital de Cuba y con la mayor densidad poblacional, aporta alrededor del $27 \%$ de los casos nuevos de tuberculosis. El presente artículo propone describir la distribución y tendencia de las tasas de incidencia de tuberculosis (1986-1998) por municipios y edades en la provincia ciudad de la Habana.

Método: Se calcularon las tasas y el porcentaje de variación total de las tasas, así como el promedio anual de variación en dos etapas y entre ellas (1986-93 y 1994-98). Las tendencias se calcularon mediante regresión lineal simple.

Resultados: De 1986 a 1993 la tasa de incidencia provincial ascendió un 6\%. De 1994 a 1998 decreció en $7.3 \%$. Los 3 municipios con mayor densidad poblacional mostraron mayor incremento en las tasas. De los 15 municipios sólo en el de La Lisa disminuyeron las tasas durante todo el período. Las tasas por edades fueron similares en todos los municipios, más altas en sujetos $\geq 65$ años. El $0.6 \%$ de los casos notificados correspondían a personas menores de 15 años. Las tasas de tuberculosis infantil fueron muy bajas en el período estudiado.

Conclusiones: Las diferencias en los municipios requieren de acciones diferenciadas para lograr mayor impacto y repercusión en el país. El descenso de las tasas observado en los últimos 3 años denota el control de la epidemia y la recuperación del programa nacional de control de la tuberculosis. dencia.

Palabras clave: Tuberculosis. Epidemiología. Ten-

Correspondencia:

Instituto de Medicina Tropical "Pedro Kourí"

Apartado Postal 601

Marianao 13

Ciudad de La Habana. Cuba

Correo electrónico: susana@ipk.sld.cu
ABSTRACT

\section{Distribution and Trend in Tuberculosis by Age Groups and Municipalities in the Province of Havana City, Cuba} (1986-1998)

Background: The province of Havana City, the capital and most densely populated city in Cuba, totals around $27 \%$ of the new cases of tuberculosis. This article is for the purpose of describing the distribution and trend of the tuberculosis cases rates from 1986 to 1998 , by municipalities and age groups in this province.

Method: A calculation was made of the rates and of the total percentage of deviation of these rates, in addition to the annual average deviation both throughout two time periods and between the two (1986-1993 and 1994-1998). The trends were calculated by simple linear regression.

Results: Throughout the 1986-1993 period, the case rate for this province underwent a $6 \%$ increase. Throughout the 1994-1998 period, it dropped by $7.3 \%$. The three most denselypopulated cities showed a higher rate increase. Of the 15 municipalities, only La Lisa showed a drop in the rates throughout the entire period in question. The rates by ages were similar throughout all of the municipalities, being higher for individuals $>65$ years of age. Six tenths $(0.6 \%)$ of the cases reported involved people under 15 years of age. The infantile tuberculosis rates were very low for the time period under analysis.

Conclusions: The differences among the municipalities in question make it necessary for different measures to be taken to achieve a greater impact and repercussion in Cuba. The drop in the rates detected over the last three years is indicative of the epidemic being brought under control and the national tuberculosis control program being reinstated.

Key Words: Tuberculosis. Epidemiology. Trend.

\section{INTRODUCCION}

La tuberculosis (TB) sigue constituyendo un problema para la salud pública a 
nivel mundial. La OMS determinó que en la década de los 90 la tuberculosis ha sido la causa más frecuente de enfermedad atribuible a un agente infeccioso en el mundo y la ha declarado una emergencia mundial $^{1}$. Anualmente enferman unos 8 millones de personas, el $95 \%$ en los países en vías de desarrollo, y mueren aproximadamente tres millones de enfermos de tuberculosis al año $0^{2,3}$.

A partir de 1959 la situación de la tuberculosis en Cuba mejoró notablemente. En 1970 se implantó y generalizó en todo el país un programa integrado en todos los servicios de salud, basado en el diagnóstico bacteriológico y el tratamiento ambulatorio controlado ${ }^{4}$. A partir de este momento la notificación de casos sufre una disminución paulatina, de una tasa de 14,3 por 100.000 habitantes en 1972 a 4,7 en $1991^{5}$.

Sin embargo, a partir del año 1992 se produce un incremento progresivo de los casos notificados en todas las provincias del país, que invirtió la tendencia descendente que existía ${ }^{5}$. En un informe reciente de la Dirección Nacional de Epidemiología del Ministerio de Salud Pública (MINSAP) sobre la situación actual del programa de tuberculosis, se corroboran los planteamientos de González y cols ${ }^{5}$, que atribuyen esta situación reemergente al incremento del riesgo de enfermar por la situación económica adversa del país y la pérdida de prioridad en las acciones de control del programa. Para contribuir al control de la enfermedad en el país es necesario conocer en detalle su situación, tanto en el ámbito local como vista en su conjunto. La provincia Ciudad de la Habana, capital del país y con la mayor densidad poblacional, aporta alrededor del $27 \%$ de los casos nuevos, por lo que en este artículo nos propusimos describir la distribución y la tendencia de las tasas de casos nuevos notificados de tuberculosis de 1986 a 1998, por grupos de edades y por municipios en la Ciudad de la Habana, a fin de esclarecer los territorios que requieren mayor priorización.

\section{MATERIAL Y MÉTODOS}

La provincia Ciudad de la Habana tiene una extensión territorial de $727,4 \mathrm{~km}^{2}$, una población estimada de 2.174 .999 habitantes y una densidad de 2990,1 habitantes por $\mathrm{km}^{2}$. Está dividida en 15 municipios, segmentados a su vez en 81 áreas de salud, existiendo un policlínico en cada una de ellas. Cada uno de los 81 policlínicos está dotado de un laboratorio clínico donde, entre otras investigaciones, se realizan los exámenes directos de esputos, en 17 hospitales se hacen baciloscopias y en tres de ellos se realizan cultivos. Completa la red de diagnóstico, la dotación de tres laboratorios territoriales de referencia y un laboratorio provincial. Las cepas de micobacterias aisladas son tipificadas en el Laboratorio Nacional del Instituto Pedro Kourí (IPK) ${ }^{6}$. Cada área de salud está dividida en sectores de población (con 700 a 800 habitantes cada uno) atendidas por un médico y una enfermera de familia. Se cuenta actualmente con 3.992 médicos en total para atender estos servicios.

En nuestro programa de control el diagnóstico de TB se establece por la identificación, aislamiento o demostración indirecta del bacilo de Koch, siendo factores determinantes para definir un caso la localización de la enfermedad, la gravedad, los resultados de la bacteriología y los antecedentes de tratamientos previos de la enfermedad. Este último resulta de gran importancia para la vigilancia epidemiológica de la resistencia y para la administración de un régimen adecuado de tratamiento ${ }^{6}$.

Una vez detectado un caso se realiza su clasificación para ultcrior cstudio y tratamiento, en dependencia de que sea caso nuevo, recaída, fracaso terapéutico, abandono o caso crónico. La localización de casos es la actividad fundamental de pesquisa que se lleva a cabo en todas las unidades del sistema nacional de salud (SNS) e instituciones penales, con el objetivo de encontrar, con la mayor rapidez, el máximo número posible de enfermos que constituyen la fuente principal de contagio, los 
casos de tuberculosis pulmonar con baciloscopia positiva (TBp BK+).

El médico consultante debe identificar, mediante búsqueda activa, organizada y sistemática, a los enfermos sintomáticos respiratorios de más de 14 días $(\mathrm{SR}+14)$, tanto en la población mayor de 15 años que acude a consulta, como en los grupos de alto riesgo. Una vez identificado el $S R+14$, el médico lo registrará e indicará dos exámenes directos de esputo y cultivo de la primera muestra útil ${ }^{6}$.

La TB es una enfermedad de declaración obligatoria, con notificación inmediata y registro de los enfermos que se diagnostiquen, lo cual es de estricto cumplimiento para todos los médicos que trabajan en el SNS. Se deben notificar todos los casos nuevos de TB que se consideren activos e inicien el tratamiento específico, así como las recaídas, llevando la tarjeta de enfermedades de declaración obligatoria (EDO, modelo 84-01), que recoge la unidad que diagnostica, provincia, semana estadística, nombre y apellidos de la persona, edad, sexo, dirección, municipio, provincia, nombre completo de la enfermedad (incluye clasificación), fecha de notificación y médico informante.

La unidad que notifica envía la información inmediatamente y posteriormente la tarjeta de EDO al Centro Provincial de Higiene y Epidemiología y de ahí al Ministerio de Salud Pública. El médico de familia, junto al jefe municipal del programa, realizan el estudio de los contactos y la encuesta epidemiológica. El médico de familia administra el tratamiento supervisado (un ciclo de 7 meses con 4 drogas en dosis únicas diarias, en la primera etapa de 8 semanas, y dos drogas dos veces por semana hasta completar 100 dosis) y realiza el seguimiento sistemático del caso.

Para este estudio un caso nuevo de tuberculosis pulmonar se definió como aquel diagnosticado y notificado por primera vez, en el cual se demostró la presencia de $M$. tuberculosis por cualquier método diagnóstico. Esto es válido para los casos notificados de 1986 a 1993, pues en el año 1994 se produjo un cambio en cuanto al criterio diagnóstico en el programa nacional ${ }^{7}$, incluyéndose dentro de los casos a notificar los que tienen examen bacteriológico de esputo negativo que antes se daban como "sospechosos", se trataban y no se notificaban. La tuberculosis extrapulmonar se definió de acuerdo con los criterios clínicos, radiológicos o histológicos de los órganos afectados?. Por tal motivo, y para efectos de un análisis correcto de los datos, dividimos el período a estudiar en dos etapas: 1986-1993 y 1994-1998, ya que rigieron diferentes criterios diagnósticos.

Los procedimientos del programa de control fueron publicados anteriormente y pueden ser leídos en diversas revistas ${ }^{5,8,9}$. Los datos sobre tuberculosis se obtuvieron del registro de notificaciones de casos nuevos del Centro Provincial de Higiene y Epidemiología de Ciudad de la Habana. Las tasas de notificación de casos nuevos se calcularon utilizando las estimaciones de la población realizadas por la Dirección Nacional de Estadísticas del Ministerio de Salud Pública, con los datos del censo y las proyecciones de la población cubana proporcionadas por el $\mathrm{Co}$ mité Estatal de Estadísticas ${ }^{10}$.

El análisis de los datos se realizó con el paquete epidemiológico y estadístico Epiinfo6, en el cual se calcularon las tasas así como las tendencias mediante regresión lineal simple. También se calcularon los porcentajes de variación total de las tasas y el promedio anual de variación en ambas etapas y entre ellas. Para el período 1994-98 se presentan también las tasas de tuberculosis pulmonar según la nueva clasificación de baciloscopia positiva $(\mathrm{BK}+)$ y negativa (BK-). Las tasas por grupos de edad para todo el período se calcularon utilizando como denominador la población media del período multiplicado por 13 .

\section{RESULTADOS}

En la primera etapa del período estudiado (tabla 1), comprendida de 1986 hasta 
1993, la notificación de casos nuevos de tuberculosis y su incidencia anual en la ciudad de la Habana, sufrió un discreto ascenso de $9,4 \times 100.000$ habitantes en 1986 a $10,0 \times 100.000$ habitante en 1993 , lo cual representa un aumento de un $6,4 \%$ durante estos 7 años a razón de un $0,9 \%$ anual como promedio, lo que hace que la rec- ta de regresión de la tendencia tenga una pendiente casi nula (figura 1). Sin embargo, este aumento se hace más notable al compararlo con el de 1991, año en que se produce la incidencia más baja en la provincia, en relación con lo cual se produjo un incremento del $30 \%$ de la incidencia anual (58 casos nuevos más).

Tabla 1

Variación porcentual de la incidencia de tuberculosis en la provincia Ciudad de La IIabana y sus municipios correspondientes a dos períodos

\begin{tabular}{|c|c|c|c|c|c|c|c|c|}
\hline \multirow{3}{*}{ MUNICIPIO } & \multicolumn{4}{|c|}{ PERIOODO 1986-1993 } & \multicolumn{4}{|c|}{ PERÍODO 1994-1998 } \\
\hline & \multicolumn{2}{|c|}{$\begin{array}{c}\text { INCIDENCIA } \\
\text { TASAS } \times 10^{5}\end{array}$} & \multicolumn{2}{|c|}{$\begin{array}{c}\text { VARIACIÓN } \\
\%\end{array}$} & \multicolumn{2}{|c|}{$\begin{array}{l}\text { INCIDENCIA } \\
\text { TASAS X10 }\end{array}$} & \multicolumn{2}{|c|}{$\underset{\%}{V A R I A C I O ́ N} *$} \\
\hline & 1986 & 1993 & GlobaL & $\begin{array}{c}\text { Media } \\
\text { anual** }\end{array}$ & 1994 & 1998 & Global & $\begin{array}{l}\text { Media } \\
\text { anual }^{* *}\end{array}$ \\
\hline PLAYA & 6.0 & 9.7 & +61.6 & 8.8 & 9.4 & 8.5 & -9.6 & 2.4 \\
\hline PLAZA & 8.8 & 11.4 & +29.5 & 4.2 & 15.7 & 7.2 & -54.1 & 13.5 \\
\hline CENTRO HABANA & 11.8 & 15.9 & +34.7 & 4.9 & 19.3 & 22.5 & +16.6 & 4.1 \\
\hline HABANA VIEJA & 11.3 & 19.1 & +69.0 & 9.8 & 22.1 & 21.9 & -0.9 & 0.2 \\
\hline HABANA DEL ESTE & 3.8 & 7.6 & +100.0 & 14.3 & 7.4 & 9.9 & +33.8 & 8.4 \\
\hline GUANABACOA & 5.4 & 4.9 & -9.2 & 1.3 & 17.3 & 35.9 & +107.5 & 26.8 \\
\hline REGLA & 11.6 & 9.3 & -19.8 & 2.8 & 40.3 & 9.0 & -77.7 & 11.1 \\
\hline S. MIGUEL PADRÓN & 7.5 & 2.9 & -61.3 & 8.7 & 2.9 & 15.9 & +448.3 & 112.0 \\
\hline CERRO & 15.0 & 16.9 & +12.6 & 1.8 & 17.1 & 15.3 & -10.5 & 2.6 \\
\hline MARIANAO & 7.1 & 7.2 & +1.4 & 0.2 & 17.0 & 18.9 & +11.1 & 2.8 \\
\hline LA LISA & 10.6 & 7.5 & -29.2 & 4.2 & 13.3 & 10.3 & -22.5 & 5.6 \\
\hline IO DE OCTUBRE & 14.3 & 10.0 & -30.0 & 4.3 & 18.8 & 10.8 & -42.5 & 10.6 \\
\hline BOYEROS & 6.0 & 3.6 & -40.0 & 5.7 & 11.4 & 12.2 & +6.5 & 1.6 \\
\hline ARROYO NARANJO & 11.8 & 5.7 & -51.7 & 7.4 & 15.8 & 13.2 & -16.4 & 4.1 \\
\hline COTORRO & 3.6 & 3.1 & -13.9 & 2.0 & 17.1 & 12.1 & -29.2 & 7.3 \\
\hline PROVINCI & 9.4 & 10.0 & +6.4 & 0.9 & 15.5 & 14.4 & -7.3 & 1.8 \\
\hline
\end{tabular}

* VARIACIÓN: - Reducción global = valor al inicio del período - valor al final del pcríodo / valor al inicio del período x 100 - aumento global = valor al final del período - valor al inicio del período $/$ valor al inicio del período $\mathrm{x} 100$ ** reducción o aumento media anual = porcentaje de reducción o aumento global / (número de años - 1) 
Figura 1

Incidencias y tendencia de la TB en la Ciudad de La Habana 1986-1993

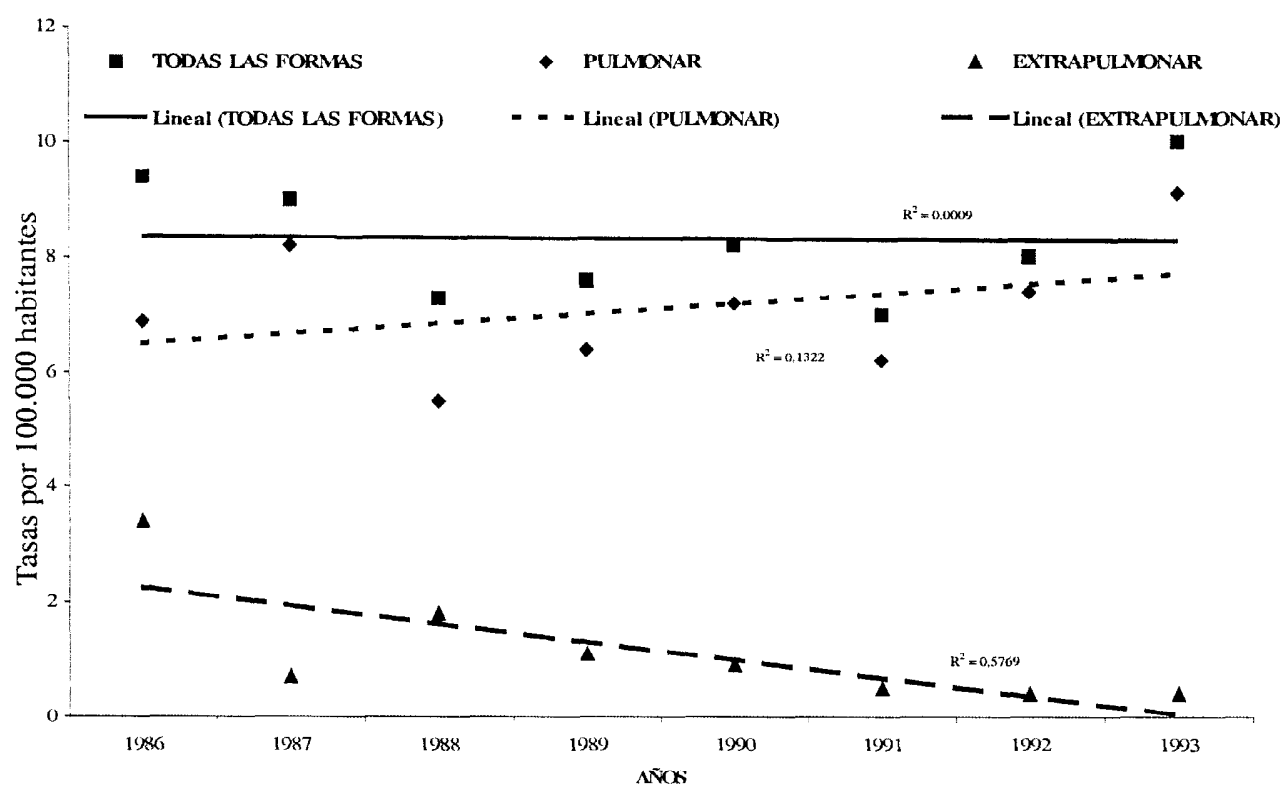

En cl año 1994, con la redefinición de casos introducida en el Programa Nacional, se produjo un incremento en la notificación de casos nuevos (135) y su respectiva incidencia anual $(15,5)$, lo que representa un aumento de $35,5 \%$ en relación con el año anterior.

Analizando por separado sus 15 municipios, obscrvamos que en el período 1986-93 tuvieron un incremento de más del $20 \%$ de sus incidencias anuales los municipios Playa, Plaza, Ccntro Habana, Habana Vieja y Habana del Este, y cifras menores de incremento Cerro y Marianao. Los restantes 8 municipios experimentaron descenso en sus tasas, siendo más marcado en San Miguel del Padrón, Arroyo Naranjo, Boyeros, 10 de Octubre y La Lisa.

De 1994 a 1998 se produjo un nuevo descenso de la incidencia de la enfermedad de $7,3 \%$ a razón de $1,8 \%$ anual en la provincia (tabla 1 y figura 2). En los diferentes municipios el comportamiento osciló desde incidencias anuales tan bajas como 2,9 en San Miguel del Padrón en 1994 hasta 40,3 en Regla en ese mismo año. Experimentaron crecimiento importante de sus incidencias en 1998 en relación con 1994 los municipios de San Miguel del Padrón y Guanabacoa, y en menor cuantía Habana del Este, Centro Habana, Marianao y Boyeros. Los restantes 9 municipios lograron un descenso de sus incidencias anuales en este período, que fue más marcado en Regla, Plaza, 10 de Octubre y Cotorro.

Es de notar que los municipios Centro Habana, Habana Vieja y Cerro se mantuvieron con cifras superiores a la media provincial durante todo el período de 13 años estudiado, correspondiendo éstos a los 3 municipios de mayor densidad poblacional de la capital (datos aportados por el Centro Nacional de Desarrollo Demográfico de Cuba). Por el contrario, municipios periféricos y con áreas semirurales, tales como Habana del Este, Lisa, Boyeros y Cotorro, se mantuvieron con las menores incidencias durante todo el período.

En la tabla 2 mostramos el comportamiento en la provincia en los últimos 5 años 
Figura 2

Incidencias y tendencia de la TB en la Ciudad de La IIabana 1994-1998

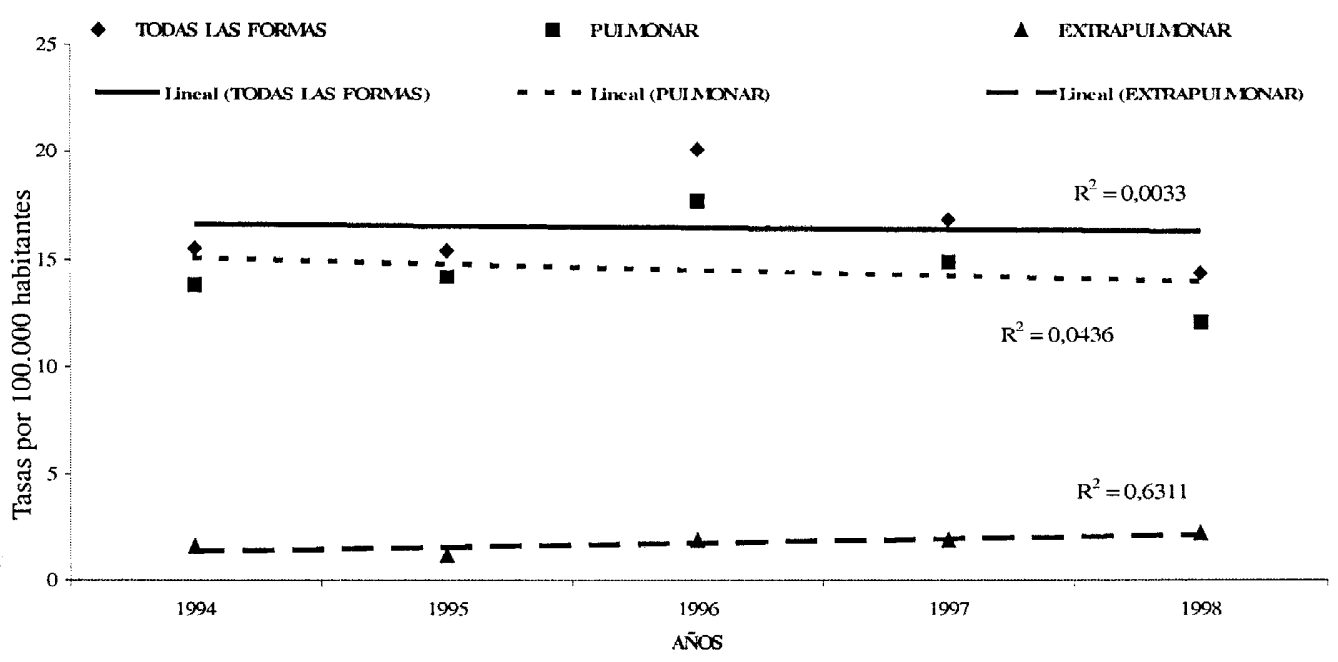

Tabla 2

Número de casos e incidencia anual de tuberculosis pulmonar (x 100000 habitantes) BK+ y BK- de 1994 a 1998 en la provincia Ciudad de La Habana.

\begin{tabular}{|l|c|c|c|c|c|c|}
\hline \multirow{2}{*}{ Año } & \multicolumn{2}{|c|}{ BK+ } & \multicolumn{2}{c|}{ BK- } & \multicolumn{2}{c|}{ TOTAL } \\
\cline { 2 - 7 } & Casos & Tasa & Casos & Tasa & Casos & Tasa \\
\hline 1994 & 254 & 11,7 & 108 & 4,9 & 362 & 16,6 \\
\hline 1995 & 231 & 10,5 & 100 & 4,6 & 331 & 15,1 \\
\hline 1996 & 262 & 11,9 & 129 & 5,9 & 391 & 17,7 \\
\hline 1997 & 208 & 9,4 & 123 & 5,5 & 331 & 14,9 \\
\hline 1998 & 178 & 80,0 & 93 & 4,1 & 271 & 12,1 \\
\hline
\end{tabular}

BK+:Baciloscopia positiva.

BK-: Baciloscopia negativa.

de la scrie según la nueva clasificación adoptada en 1994. La tasa descendió tanto en los sujetos con $\mathrm{BK}+$ como en aquellos con BK-.

Las incidencias anuales por grupos de edades se comportaron de forma similar en todos los municipios, con cifras mucho más altas en el grupo de 65 y más años al que correspondió el $36,1 \%$ de los casos de la provincia en todo el periodo. En 6 municipios (Habana Vieja, Cerro, Centro Habana, San Miguel del Padrón, Plaza y Boyeros) la incidencia para este grupo de edad fue superior a la de la provincia, y la más baja fue en regla 
con 4,1/100 000 habitantes. En el grupo de niños de 0 a 4 años no hubo tuberculosis de 1986 a 1994; de 1995 a 1998 se produjeron algunos casos, siendo la incidencia más elevada en 1996 con 3,4x $10^{5}$. En las edades de 5-14 años también se observaron incidencias muy bajas, por debajo de $1 \times 10^{5}$ en todo el período analizado.

\section{DISCUSIÓN}

El cambio producido en la definición de $\operatorname{casos}^{7}$ trajo consigo un aumento en las notificaciones que explica, en parte, el brusco incremento del número de casos a partir de 1994, aunque ya desde 1992 se habían venido produciendo incrementos en algunos municipios que, al igual que en el resto del país, ha sido atribuido al recrudecimiento de la crisis económica, la disminución relativa de la prioridad de la tuberculosis en el conjunto de los problemas de salud del país, la reducción de algunos recursos necesarios, cambios en la organización de las medidas de control, la disminución del estado de alerta y de la conciencia sobre el riesgo de infección, la interacción de la tuberculosis con la infección por el VIH, y el debilitamiento general de la cantidad y la calidad de las acciones de localización de $\operatorname{casos}^{5}$ (informe del MINSAP).

No obstante este aumento, la incidencia anual de tuberculosis en nuestra capital durante este período es inferior a las del resto de los países de América Latina, teniendo además en cuenta las diferencias en la operación de la vigilancia y control en estos países. En el período 1988-89 se registró una incidencia anual media de $42 / 100.000$ en la región ${ }^{11}$ y en 1994 ésta se calculó en $34,9 / 100000^{12}$. En los Estados Unidos la incidencia de tuberculosis también experimentó un aumento de $9,4 \times 100.000$ habitantes en 1984 a 10,5 en 1992, encontrándose las incidencias más elevadas del país en las ciudades de más de 250.000 habitantes, con cifras de hasta más de $60 \times 100.000^{13}$. A partir de 1993 comenzaron a descender y ya en 1998 fueron de $6,8 \times 100.000^{14}$.
En los últimos 50 años se ha producido un éxodo masivo de población desde las áreas rurales a las urbanas en muchos países, de lo cual no ha estado exento el nuestro. Esta rápida expansión de los centros urbanos ha creado muchos problemas higiénico-sanitarios y de hacinamiento, incrementándose las probabilidades de la transmisión de enfermedades infecciosas como la tuberculosis $^{15}$; no obstante, en nuestra ciudad capital la incidencia anual de esta enfermedad está muy por debajo de la del resto de las grandes ciudades de América, incluido Estados Unidos ${ }^{16}$ y se comporta de forma similar al resto de las provincias del país (informe del MINSAP) ${ }^{5,7}$. Como causa de morbilidad la tuberculosis ocupa el sexto lugar entre las enfermedades transmisibles en la capital (datos no publicados).

En la ciudad de Nueva York la incidencia de tuberculosis es 4 veces el promedio nacional, mientras que en Harlem es 20 veces más alta. A pesar de que Nueva York cuenta con el 3\% de la población de Estados Unidos, en 1994 registró el $12 \%$ de todos los casos de tuberculosis del país ${ }^{16}$ y en 1998 el 8,5\% de éstos $^{14}$. En 1997 la incidencia de TB en Dinamarca fue $7,5 \times 10^{5}$ con un ascenso del $86 \%$ en relación a 1986, principalmente debido a la inmigración procedente de países de alta prevalencia, pero en la capital, Copenhagen, se observó un incremento entre hombres marginados de $35-55$ años de edad ${ }^{17}$. En Haití, el país más pobre de América, a pesar de que el $70 \%$ de los casos de tuberculosis no se notifican, se estima una incidencia anual superior a los 500/100.000 en las zonas urbanas y de $90 / 100000$ en las rurales ${ }^{18}$. Mientras en África, en un estudio en Tanzania, se encontró un incremento de la TB del $200 \%$ de 1985 a 1995 y en 1997 la tasa fue de $111 \times 10^{5}$ en la región de Mwanza ${ }^{19}$. Etiopía registró una incidencia de $155 \times 10^{5}$ en $1996^{20}$.

En cuanto a la distribución por edad, el predominio en ancianos se corresponde con el del resto del país, y coincide con lo registrado en países desarrollados con un control adecuado de la enfermedad, lo que habla a 
favor de las reactivaciones endógenas como posible causa fundamental del incremento de la enfermedad, pues se mantiene muy baja la incidencia en niños y jóvenes. Aunque no se han hecho estudios sobre la evolución del riesgo de infección tuberculosa en nuestra población, su estimación a partir de los casos nuevos con baciloscopia positiva en Ciudad de la Habana sería de alrededor de $0,2 \%{ }^{21}$. En este aspecto puede haber influido la alta cobertura del tratamiento antituberculoso. Son éxitos también de nuestro programa la eficiencia y eficacia del tratamiento, que se han mantenido durante este período por encima de 85 y $97 \%$ respectivamente. Así mismo no se han reportado cifras de resistencia a medicamentos antituberculosos mayores de $5 \%$ en estos años (datos no publicados del Laboratorio Nacional de Referencia de Micobacterias del Instituto "Pedro Kourí"). La vacunación con $B C G$ en recién nacidos, que en 1998 fue de $98,8 \%$, cifra similar a la registrada en el resto de los años de la serie, podría haber contribuido también en la desaparición de las formas clínicas graves de la tuberculosis infantil.

Como base de estos éxitos está la existencia durante más de 25 años de un programa de control basado en el DOTS y la temprana localización de casos. Desde 1963 se puso en marcha el primer programa de control organizado, aún basado en dispensarios y hospitales antituberculosos, con ampliación de las acciones de control, que se tradujeron en cambios apreciables de los indicadores epidemiológicos de la enfermedad ${ }^{22}$. En 1971 se puso en marcha en todo el país, un programa integrado por todos los servicios de salud, basado en el diagnóstico bacteriológico y el tratamiento ambulatorio controlado ${ }^{4}$. Como consecuencia de la aplicación de este programa se produjo un descenso de la incidencia de TB a un ritmo de $5 \%$ anual hasta 1991.

La diferencia de comportamiento observada en los municipios tal vez puede explicarse por el mejor funcionamiento del programa en algunos de ellos sin perder de vista las influencias de sus condiciones socioeconómicas y geográficas diferenciales. Tal es el caso de La Lisa, municipio periférico con una parte semirural y poca densidad poblacional en comparación con el resto, así como un estable trabajo en la atención primaria de salud.

Podemos concluir que en el periodo estudiado la tendencia total fue ligeramente ascendente en la provincia y, como tal, cn sus municipios, excepto en La Lisa. El comportamiento fue diferente en los municipios, por lo que el programa necesita de acciones diferenciadas en cada uno de ellos para lograr que disminuyan los casos en la capital y por tanto en el país, por aportar ésta alrededor del $27 \%$ de las notificaciones. El descenso observado en la incidencia de TB en los últimos 3 años, tanto en la capital como $\mathrm{cn}$ cl resto del país, es expresión del control de la epidemia y la recuperación del Programa Nacional de Control de la Tuberculosis.

\section{BIBLIOGRAFÍA}

1. OPS/OMS. Programa de OMS contra la TB: Marco para el control eficaz de la tuberculosis. WHO/TB94.179(s).

2. Galdós-Tangüis H, Caylà J. Tuberculosis infantil. Protocolo de trabajo para su diagnóstico y control en los países de alta prevalencia. Ajuntament de Barcelona; 1995. p. 9.

3. Raviglione MC, Snider DE, Kochi A. Global epidemiology of TB. morbidity and mortality of a worldwide epidemic. JAMA 1995; 273 (3):220-5.

4. República de Cuba, Ministerio de Salud Pública, OPS. Documento base. La Habana. MINSAP. Programa nacional de control de la tuberculosis; 1970.

5. González E, Armas L, Machín A. Tendencias por provincias de la tuberculosis en Cuba: 1979-1993. Bol Oficina Sanit Panam 1995; 119(5):396-404.

6. Ministerio de Salud Pública. Programa nacional de control de la tuberculosis: manual de normas y procedimientos. MINSAP. La Habana: ECIMED; 1999.p.13-14. 
7. Ministerio de Salud Pública. Actualización del programa nacional de control de la tuberculosis. Documento. Dirección Nacional de Epidemiología; 1995. p. 3-5.

8. González E, Armas L, Alonso A. Tuberculosis in the Republic of Cuba: its possible elimination. Tuberc Lung Dis 1994; 75:188-94.

9. Marrero A, Carreras L, Santín M. El programa de control de la tuberculosis en Cuba. Rev Cub Med Gen Integral. 1996; 12(4):381-5.

10. República de Cuba. Comité estatal de estadísticas. Instituto de demografía y censo. Censo de población y viviendas 1981. La Habana: Comité Estatal de Estadísticas, Instituto de Demografía y Censo; 1984.

11. Sudre P, Ten Dam G, Chan C, Kochi A. Tuberculosis in the present time: a global overview of the tuberculosis situation. WHO/TUB/91.158. Gcneva: WHO; 1991.

12. WHO. Tuberculosis notification update. Weekly Epidemiol Record. 1996; 71 (9):65-69.

13. Centers for Disease Control and Prevention. Prevention and control of tuberculosis in USA. Comunities with at-risk minority populations; and prevention and control of tuberculosis among homeless persons. MMWR 1992; 41(rr-s).

14. CDC. Morbilidad por tuberculosis en los Estados Unidos. MMWR 1999; 48 (33):732-5.

15. Bosman MC. Tuberculosis in big cities. What more do we need to know? Conference on glo- bal lung health and the 1996 annual meeting of the IUATLD. Paris, France. 2-5 october. 1996. Tuberc Lung Dis 1996; 77 (suppl 2): 17.

16. Menzies D. Tuberculosis in big cities. the urban factor in tuberculosis. Conference on global health and the 1996 annual meeting of the IUATLD. Paris, France. 2-5 october 1996. Tuberc Lung Dis 1996; 77 (suppl 2): 16.

17. Lilleback T, Poulsen S, Kok-Jensen A. Tuberculosis treatment in Denmark: treatment outcome for all Danish patients in 1992. Int J Tuberc Lung Dis 1999; 3 (7):603-12.

18. Hypolite PR, Pope JW. Infección por VIH y tuberculosis en Haití. Bol Oficina Sanit Panam. 1995; 118 (2):161-9.

19. Wandwalo ER, Morkve O. Delay in tuberculosis case finding and treatment en Mwanza, Tanzania. Int J Tuberc Lung Dis 2000; 4 (2):133-8.

20. Getahun H, Maher D. Contribution of "TB clubs" to tuberculosis control in a rural district in Ethiopia. Int J Tuberc Lung Dis 2000; 4 (2): 174-8.

21. Styblo K. The relationship between the annual risk of tuberculosis infection and the risk of developing infectious tuberculosis. Bull Int Union Against Tuberc 1985; 60:117-19.

22. Grupo Nacional dc Tuberculosis. Evolución de la situación epidemiológica de la tuberculosis en Cuba de 1959 a 1969. Bol Hig Epidemiol 1972; 10:41-52. 


\section{CORRECCIONES}

En el trabajo Distribución y tendencia de la tuberculosis por grupos de edades y por municipios en Ciudad de la Habana, Cuba. (1986-1998). S Borroto Gutiérrez, L Armas Pérez. CE González Ochoa, O Peláez Sánchez, AL Arteaga Yero y Sevy Court. Rev Esp Salud Pública 2000; 74: 507-515, se deben corregir las siguientes erratas:
En los nombres de los autores donde dice Luis Armas Pérez debe decir Luisa Armas Pérez.

En la tabla 2 de la página 512, en la tasa de $\mathrm{BK}+$ del año 1998 donde dice 80,0 debe decir 8,0 . 